\title{
संगीत में बन्दिश का महत्व
}

\author{
डॉ. श्रुति होरा
}

Associate Professor (Music Instrumental), PGGCG, Sector -11, Chandigarh

\section{सार-संदर्भ}

बन्दिश से अर्थ-भाव बाँधाने की क्रिया से है जिसमें पहले से रचा गया प्रबन्ध गीत, कविता आदि की शब्द योजना तथा संगीत में शब्दों के साथ-साथ स्वरता योजना का भी समावेश होता है। शास्त्रीय संगीत में बन्दिश ही सम्पूर्ण राग-विस्तार का आधार बनती है। बन्दिश के अंग-भारतीय संगीत में भी किसी रचना के मूलतत्व-स्वर, लय तथा ताल माने जाते हैं। गायन अथवा वादन प्रस्तुतीकरण में राग विस्तार के अनेक आवश्यक तत्व होते हैं जैसे नाद, श्रुति, स्वर, वर्ण, अलंकार, आलाप, तान, गमक, स्थायी इत्यादि सूक्ष्म रूप से देखने पर ऐसा ज्ञात होता है कि राग वे अन्तर्गत प्रयुक्त समस्त तत्वों की प्रस्तुति एक ढाँचे के अन्तर ही होती है। बन्दिश की स्वर रचना करते समय राग के वर्ज्य-अवर्ज्य, अल्प-बहुत्व, वादी-संवादी आदि स्वरों तथा राग स्वरूप का पूर्ण ध्यान अनिवार्य है। उच्च कोटि की बन्दिश रचना के लिए संगीत शास्त्र तथा साहित्य शास्त्र का यथेष्ट ज्ञाता होना आवश्यक है। कुंजी शब्द : बन्दिश, घराना, संगीत।

श्री गुलाम रसूल द्वारा लिखित एक लेख के अनुसार बन्दिश से अर्थ-भाव बाँधाने की क्रिया से है जिसमें पहले से रचा गया प्रबन्ध गीत, कविता आदि की शब्द योजना तथा संगीत में शब्दों के साथ-साथ स्वरता योजना का भी समावेश होता है। शास्त्रीय संगीत में बन्दिश ही सम्पूर्ण राग-विस्तार का आधार बनती है अर्थात् बन्दिश के माध्यम से कलाकार, आलाप तान आदि विविध अंगो द्वारा राग का विस्तार करता है। बन्दिश के अंग-भारतीय संगीत में भी किसी रचना के मूलतत्व-स्वर, लय तथा ताल माने जाते हैं। बन्दिश के अधिकांशतय तथा यह दो अंग ही माने जाते हैं। ध्रुपद, धमार शैलियों के संचारी और आभोग नामक दो शेष अंगों का प्रचार आज नाम मात्र है। उच्च कोटि की बन्दिश रचना के लिए स्वीकार का संगीत शास्त्र तथा साहित्य शास्त्र का यथेष्ट ज्ञाता होना आवश्यक है। बन्दिश में प्रयुक्त शब्द, सरल एवं भाव-व्यंजक होने के साथ-साथ लयबद्ध होना चाहिए। बन्दिश की स्वर रचना करते समय राग के वर्ज्य-अवर्ज्य, अल्प-बहुत्व, वादी-संवादी आदि स्वरों तथा राग स्वरूप का पूर्ण ध्यान अनिवार्य है। उस्ताद अपने शिष्यों को किसी राग का ज्ञान करवाने के लिए सर्वप्रथम बन्दिश सिखाते हैं।

\section{संगीत में बन्दिश का महत्व}

संगीत में बन्दिश शब्द से अभिप्राय ऐसी रचना से है। जिसमें इस कला के स्वर और ताल आदि से संबधित नियमों का पालन किया जाता है। डा॰ सुभद्रा चौधरी ने बन्दिश का अर्थ पूर्व रचित गीत बताया है। क्योंकि इसकी रचना पहले ही की जाती है। श्री गुलाम रसूल द्वारा लिखित एक लेख के अनुसार बन्दिश से अर्थ-भाव बाँधाने की क्रिया से है जिसमें पहले से रचा गया प्रबन्ध गीत, कविता आदि की शब्द योजना तथा संगीत में शब्दों के साथ-साथ स्वरता योजना का भी समावेश होता है। इस आधार पर यह कहा जा सकता है कि 'बन्दिश' स्वर, लय व ताल आदि पर आधारित होती है। जिसका स्वरूप पहले से ही सुनियोजित होता है। अपनी व्यक्तिगत रूचि, कल्पना अथवा भावाभिव्यक्ति की दृष्टि से उत्तर भारत के कलाकार इसमें थोड़ा बहुत परिवर्तन कर लेते हैं, किन्तु यह परिवर्तन केवल एक सीमा तक ही स्वीकारणीय होता है अब तक बन्दिश के मूल स्वरूप अथवा ढाँचे पर कुठाराघात नहीं होता। 
शास्त्रीय संगीत में बन्दिश ही सम्पूर्ण राग-विस्तार का आधार बनती है अर्थात् बन्दिश के माध्यम से कलाकार, आलाप तान आदि विविध अंगो द्वारा राग का विस्तार करता है। बन्दिश किसी घराने की परम्परागत शैली का एक महत्वपूर्ण अंग मानी जाती है। उसकी शेलीगत विशेषताएं स्थायी रूप से उसकी बन्दिशें से ही परिलक्षित होती है और इसीलिए किसी भी घराने पर होन वाले अध्ययन में उसकी विशिष्ट भूमिका अत्यन्त महत्वपूर्ण मानी जाती है।

बन्दिश के अंग-भारतीय संगीत में भी किसी रचना के मूलतत्व-स्वर, लय तथा ताल माने जाते हैं। गायन में गीत की कविता तथा वादन में किसी वाद्य विशेष के पाटाक्षरों अथवा सहायक बोलों का समावेश होता है। मोड़, कण, गमक, खटका, मूर्की, कृन्तन तथा विविध लयकारिणी आदि उपकरण इनके सहायक के रूप में प्रयुक्त होकर इन्हें अलंकृत करने का कार्य करते है। इनके अतिरिक्त लगभग प्रत्येक रचना, मूलतः स्थायी और अंतरा इन दो भागों अथवा अंगों पर आधारित होती है जिनका संक्षिप्त विवेचन निम्नानुसार है।

स्थायी:- यह गीत अथवा गत का प्रथम भाग होता है। इसकी स्वर रचना साधारणतः मध्य व मन्द्रं सप्तक में होती है, परन्तु कभी कभी राग अनुसार इसमें परिवर्तन भी होता है जैसे सोहनी, अड़ाना, बहार आदि रागों में स्थायी की स्वर रचना में भी मध्य व तार सप्तकों के स्वर प्रयुक्त होता है।

अन्तरा:- बन्दिश का वह भाग जो कि स्थायी से कुछ अन्तर लिये हुए होता है अन्तरा कहलाता है इसकी स्वर रचना मध्य व तार सप्तकों पर आधारित होती है।

इस प्रकार आज बन्दिश के अधिकांशतय तथा यह दो अंग ही माने जाते हैं। ध्रुपद, धमार शैलियों के संचारी और आभोग नामक दो शेष अंगों का प्रचार आज नाम मात्र है। तंत्रीकारी में कुछ कलाकार स्थायी की दूसरी पंक्ति को 'मंझार' और इसी प्रकार अन्तरे की दूसरी पंक्ति को 'आमद' भी कहते हैं।

बन्दिश के सिद्धान्त-व्यापक रुप से संगीत में उत्तम बन्दिश सम्बन्धित मान्य-सिद्धान्तों पर मूलतः दो पक्षों से विचार करना बाहूनीय है-बन्दिश रचना की दृष्टि से और उसके प्रस्तुतिकरण की दृष्टि से

बन्दिश रचना की दृष्टि से- उच्च कोटि की बन्दिश रचना के लिए स्वीकार का संगीत शास्त्र तथा साहित्य शास्त्र का यथेष्ट ज्ञाता होना आवश्यक है। बन्दिश में प्रयुक्त शब्द, सरल एवं भाव-व्यंजक होने के साथ-साथ लयबद्ध होना चाहिए। बन्दिश की स्वर रचना करते समय राग के वर्ज्य-अवर्ज्य, अल्प-बहुत्व, वादी-संवादी आदि स्वरों तथा राग स्वरूप का पूर्ण ध्यान अनिवार्य है। राग के प्रमुख स्वर समूहों का बन्दिश में कलात्मक और भावपूर्ण ढंग से प्रयोग होना चाहिए क्योंकि राग की सारी रजंकता राग-वाचक स्वर समूहों पर ही केन्द्रित रहती है और इसलिए इन स्वर समूहों पर ही केन्द्रित रहती है और इसलिए इन स्वर समूहों का प्रयोग गीत को आकर्षित बनाता है। शास्त्रीय संगीत में उत्तम बन्दिश की प्रमुख कसौटी यह भी मानी जाती है कि उसे गाते ही राग का स्वरूप स्पष्ट हो जाए और इसकी पूर्ति का एकमात्र साधन राग के प्रमुख स्वर-समूहों के प्रयोग पर निर्मित है।

बन्दिश की स्वर रचना उसी ताल में होनी चाहिए जो कि कविता के छन्द के सर्वाधिक अनुकूल है। विलम्बित लय की रचनाओं में यह नियम कुछ शिथिल पड़ता है। वस्तु वहाँ भी गति के महत्वपूर्ण शब्दों अथवा अंशो का ताल को खाली भरी की प्रमुख मात्राओं पर आज्ञा प्रभावोत्पादक होती है। 
गत या गीत का प्रारम्भ किस स्वर से किया जाये, ताल का प्रथम आवर्तन कहाँ समाप्त हो अधिक आवर्तनों के बन्दिश का अंतरा आदि भाग कहाँ से प्रारम्भ हो, बन्दिश के एक भाग से दूसरे भाग का संबंध किस प्रकार स्थापित हो जिससे कि सम्पूर्ण रचना का गठन व्यवस्थित हो, आदि स्थलों पर रचनाकार का ध्यान आवश्यक होता है।

बन्दिश की स्वर रचना करते समय अलंकार प्रयोग, राग-स्वरूप तथा कविता के भाव के अनुकूल होना चाहिए। जैसे:- दरबारी में भक्ति तथा गम्भीर भाव-युक्त रचना को स्वरबद्ध करते समय उसमें यथा स्थान मींड, कण, आन्दोलन आदि का प्रयोग श्रेयाकार होता है तथा राग बहार में चंचल भावों की रचना के लिए गीत और कण के साथ खटका, मुर्की आदि अलकरणों का प्रयोग उपयुक्त होता है। इन सिद्धान्तों के यथायोग्य एवं यथेष्ट प्रयोग से जो भी रचना की जाती है, वह सुमधुर एवं रसात्मक होती है।

बन्दिश के उत्तम प्रस्तुतिकरण की दृष्टि से- बन्दिश की रचना से भी अधिक महत्वपूर्ण इसका उत्तम रीति से प्रस्तुतिकरण है। एक योग्य कलाकार अपने विशिष्ट गुणों द्वारा साधारण रचना को भी प्रभावशाली बना सकता है। इसके प्रस्तुतीकरण में प्रथम बन्दिश का चयन समय व परिस्थिति का श्रोताओं की रूचि के अनुकूल होना अनिवार्य है। शब्दोंच्चारण, स्वर लगाव, मुख मुद्रा, हाव-भाव प्रदर्शन आदि का ध्यान भी आवश्यक होता है। लगाव जितना मर्मस्पर्शी होता है, उसी के अनुरूप राग व बन्दिश दोनों की भाव-प्रवीणता बढ़ती है। रचना के भाव, शैली और राग के अनुसार गायक को सूक्ष्म स्वर लगाव, मुर्की, गमक आदि के साथ-साथ काकु और नाद सौन्दर्य के अन्य सूक्ष्म अलंकरणों द्वारा यथायोग्य रूप से आभूषित करना चाहिए। किसी राग की बन्दिश में विलम्बित तथा द्रुत रचना में भाव-सभ्यता आवश्यक है। बन्दिश की एक पंक्ति को अनेक प्रकार से गाते हुए विविधता का संचार किया जाता है।

विलम्बित ख्याल आदि के गायन में पूरे गीत की लय के स्पष्ट दर्शन केवल स्थायी और अंतरे के मुखड़े में ही होते हैं, किन्तु सौद्धान्तिक दृष्टी से यह उचित है कि जहाँ तक हो सके बन्दिश के शेष भाग में ही ताल के प्रवाह से बन्दिश के शब्दों का संबंध पूर्णता विच्छिन्न प्रतीत न हो। दूसरे शब्दों में विलम्बित लय में भी बन्दिश को ताल की मूल लय से पूर्ण रूप से अलग नहीं होने देना चाहिए। सामान्य रूप से भले ही यह सिद्धान्त गायकों को अप्रिय और अनावश्यक बाधा में डालने वाला प्रतीत होगा किंतु व्यवहारिक रूप में अनुसारित किए जाने पर यह बन्दिश की रजंकता में वृद्धि करेगा।

किसी विशिष्ट राग में गायन के नियमित बन्दिश के चयन के समय यह ध्यान आवश्यक है कि काव्य और राग में स्पष्ट विरोध न हो। बन्दिश की रचना और प्रस्तुतिकरण संबंधी उपरोक्त सिद्धान्तों का योग्य रीति से पालन होने पर जहाँ उत्तम बन्दिश का निर्माण तो संभव ही है साथ-साथ ही गायन के गीत तथा वादन के गत में ऐसी बन्दिश के माध्यम से किसी भी राग के यथोचित प्रस्तुतीकरण तथा प्रभावोत्पादकता में सक्षमता आती है।

राग में बन्दिश का महत्व- उत्तर भारतीय संगीत में बन्दिश राग के प्रस्तुतीकरण में वह भाग होता है जिसमें स्वर और ताल दोनों का संतुलन होता है। उर्दू भाषा में कम्पोज़िशन के लिए 'बन्दिश' शब्द का प्रयोग किया गया है जो कि निबद्ध का शब्दांश अनुवाद है। जिस प्रकार शब्द के खण्डों को 'धातु ' 
कहते थे उसी प्रकार बन्दिश के खण्डों को 'तुर्क' कहा जाता है आज भी कुछ घरानेदार लोग पूरी बन्दिश के लिए अस्थाई शब्द का प्रयोग करते हैं। बन्दिश विभिन्नताओं का बुना हुआ स्वरूप है जिसमें राग के सभी पक्षों की पूर्णता है। बन्दिश सजृनात्मक प्रवृति अर्थात् बढ़त करने की क्रिया में मार्गदर्शन करती है। बन्दिश राग स्वर समूह के लिए आवर्तन का काम करती है। राग के अन्तर्गत बन्दिश को बार-बार दोहराया जाता है परन्तु फिर भी राग रजंकता में कोई विधन नहीं पड़ता। इसकी उपमा हर सुबह उदय होने वाले तथा हर शाम को अस्त होने वाले सूर्य से जा सकती है जो कि नित्यप्रति समान क्रिया है परन्तु फिर भी हर रोज़ नया रूप लेकर आता है।

गायन अथवा वादन प्रस्तुतीकरण में राग विस्तार के अनेक आवश्यक तत्व होते हैं जैसे नाद, श्रुति, स्वर, वर्ण, अलंकार, आलाप, तान, गमक, स्थायी इत्यादि सूक्ष्म रूप से देखने पर ऐसा ज्ञात होता है कि राग वे अन्तर्गत प्रयुक्त समस्त तत्वों की प्रस्तुति एक ढाँचे के अन्तर ही होती है। जिसे 'बन्दिश' कहा जाता है। बन्दिश का महत्व इस बात से भी स्पष्ट होता है कि कुछ उस्ताद अपने शिष्यों को किसी राग का ज्ञान करवाने के लिए सर्वप्रथम बन्दिश सिखाते है और तब राग का स्वरूप समझते हैं दूसरी ओर ऐसा भी देखने में आता है कि कुछ विद्यार्थी अपनी शिक्षा शासत्रीय ग्रन्थों में उपलब्ध बन्दिशों के आधार पर ही ग्रहण करते हैं। यद्यपि ये शिक्षा-ग्रहण का उचित ढंग नहीं माना जा सकता है परन्तु ग्रथों में बन्दिश प्राप्त होने से किसी राग के स्थूल स्वरूप का ज्ञान आवश्यक हो जाता है। दोनों ही स्थितियों में 'बन्दिश' का महत्व प्रत्यक्ष है।

संदर्भ

कुमार डॉ शोभित (2012), सितार बन्दिश सौन्द्रय

लालमणि मिश्र(1973), भारतीय संगीत वाद्य

संगीत विषारद .......संगीत कार्यालय हाथरस लमंत 2013

वाद्य वादन अंक....... संगीत कार्यालय हाथरस लमंत 1975

सितार वादन कला ....डॉ. शिलपी नाहर लमंत रू 2015 